\title{
Nottingham Clinico-Pathological Response Index (NPRI) after neoadjuvant chemotherapy (Neo-ACT) accurately predicts clinical outcome in locally advanced breast cancer
}

\section{Tarek M Abdel-Fatah ${ }^{1}$, Graham Ball2 ${ }^{2}$, Andrew HS Lee ${ }^{3}$, Sarah Pinder ${ }^{4}, \mathbf{R}$ Douglas MacMilan ${ }^{5}$, Eleanor Cornford 6 , Paul M Moseley ${ }^{1}$, Rafael Silverman', James Price ${ }^{1}$, Bruce Latham ${ }^{8}$, David Palmer ${ }^{8}$, Arlene Chan ${ }^{7}$, Ian O Ellis ${ }^{3}$, Stephen YT Chan ${ }^{1 *}$}

${ }^{1}$ Clinical Oncology Department, Nottingham University Hospitals, Nottingham NG51PB, UK.

${ }^{2}$ School of Science and Technology, Nottingham Trent University, Clifton campus, Nottingham NG11 8NS, UK

${ }^{3}$ Histopathology Department, Nottingham University Hospitals NHS Trust, Nottingham NG51PB, UK.

${ }^{4}$ School of Medicine, Department of Research Oncology, King's College London, Thomas Guy House, Guy's, London, SE1 9RT, UK.

${ }^{5}$ Surgical Department, Nottingham University Hospitals, Nottingham NG51PB, UK.

${ }^{6}$ Radiology Department, Nottingham University Hospitals, Nottingham NG51PB, UK.

${ }^{7}$ Curtin Health Innovation Research Institute, Curtin University, 146 Mounts Bay Rd,

Perth, Western Australia, 6000, Australia

${ }^{8}$ Western Diagnostics, Peth, Western Australia, 6000, Australia.

There are no conflicts of interest to disclose.

* Primary Corresponding author:

Dr Stephen YT Chan

Clinical Oncology Department

University of Nottingham City Hospital NHS Trust

Nottingham NG51PB, U.K.

Telephone: +44(0)1159691169 Ext: 57298

Fax: $+44(0) 1159628047$

E-Mail: steve.chan@nuh.nhs.uk 


\section{CONTEXT AND CAVEATS}

At present, there are no effective prognostic tools to guide adjuvant therapy based on response to neoadjuvant chemotherapy for locally advanced breast cancer (LABC). In the current study, a comprehensive clinico-pathological evaluation of a cohort of patients from a single centre was reviewed, including paired prechemotherapy diagnostic tumour core biopsy and post-chemotherapy surgical specimens, and a new clinico-pathological response index (NPRI) was developed. Patients can be divided into four distinct prognostic groups based on disease free survival (DFS) and breast cancer specific survival (BCSS), and the index was validated in an internal and external independent cohorts.

Patients with higher NPRI scores showed statistically significant associations with shorter DFS and BCSS. The specificity and sensitivity of NRPI is superior to currently used indexes including pathological complete response $(p C R)$, residual cancer burden and pathological TNM staging (yp-TNM). For example, the good prognosis groups included $52 \%$ of all patients based on NPRI scores, compared to only $15 \%$ of patients by using the pCR criteria. This score has the potential to become the best prognostic tool currently to determine adjuvant-therapy following neoadjuvant chemotherapy, and to standardized important factors for the reporting of results in this setting. However, the NPRI has to be tested as a clinical tool to guide, and make a difference to, the choice and outcome of adjuvant-therapy in a prospective clinical trial. 


\section{Abstract}

Purpose: There is a need to identify more sensitive clinico-pathological criteria to assess the response to Neo-ACT and guide subsequent adjuvant-therapy. Experimental Design: We performed a clinico-pathological assessment of 427 patients who had completed Neo-ACT for locally advanced breast cancer (LABC) with a median follow-up of 5-years. Patients were divided into a training set treated with anthracycline combination chemotherapy (AC, $n=172)$; an internal validation set treated with $A C$ and taxane $(n=130)$; and an external validation set treated with $A C$ with or without taxane $(n=125)$.

Results: A multivariate Cox regression model demonstrated the absence of fibrosis, presence of lympho-vascular invasion, and increasing number of lymph node metastases were significantly associated with short disease-free survival (DFS) and breast-cancer specific survival (BCSS, $p<0.01$ ), whilst reduction of tumour size was associated with DFS $(p=0.022)$. Nottingham Clinico-Pathological Response Indexes (NPRIs) were calculated and four prognostic groups (NPRI-PGs) were identified. Patients in prognostic group 2 (NPRI-PG2) for DFS ( $n=63 / 172 ; 36.6 \%)$ and BCSS (66/172; 38.4\%) have the same prognosis as those who achieved pCR (NPRI-PG1; 15\%). Receiver operating characteristic (ROC) curves indicated that the NPRI outperformed the currently used prognostic factors and adding NPRI improved their performance as a predictor for both DFS (AUC $=0.87)$ and $B C S S(A \cup C=0.88)$. Conclusions: The NPRI predicts DFS and BCSS, with a higher sensitivity than pCR. The NPRI can also improve the sensitivity and specificity of clinico-pathological response as a study end-point, for assessing response to Neo-ACT, and can serve as a valuable tool for the discovery of future predictive molecular markers. 
Key Words: Breast Cancer, Response to Neoadjuvant Chemotherapy, Clinicopathological assessment, NPRI

Running title: NPRI predicts clinical outcome in LABC after Neo-ACT 


\section{Introduction}

A meta-analysis combining data from over 3,900 patients ${ }^{1}$ with locally advanced breast cancer (LABC) demonstrated no difference in overall survival and disease progression between neoadjuvant and adjuvant chemotherapy. Several clinical trials have confirmed the safety and efficacy of neoadjuvant chemotherapy and established its utility in the management of $\mathrm{LABC}^{2}$. Although response to neoadjuvant chemotherapy may provide some indication about the potential response of the tumour to further treatment ${ }^{3}$, and may be informative about cancer biology ${ }^{4}$, the optimal method of assessing response and it impact on survival is yet to be established and there are no universally accepted criteria ${ }^{5}$.

Many studies have shown that achieving pathological complete response ( $p C R)$ after neoadjuvant chemotherapy predicts overall survival, which is independent of treatment regimen ${ }^{6-8}$. However, selected trials comparing different neoadjuvant chemotherapy regimens have failed to demonstrate an association between $\mathrm{pCR}$ rate and improved outcome ${ }^{9}$. Furthermore $\mathrm{pCR}$ is an imperfect surrogate for clinical outcome, given that: i) only a small fraction of neoadjuvant chemotherapy patients achieve pCR (3-28\%; according to the definition of pCR and chemotherapeutic regimen), ii) patients who do not achieve $\mathrm{pCR}$ may still have a good prognosis whilst patients who achieve pCR can still experience recurrent disease ${ }^{10}$, and iii) the presence of residual cancer cells observed after neoadjuvant therapy reflects a wide range of responses from near-pCR to complete resistance.

In this study we identified clinico-pathological criteria that could assess and grade response to neoadjuvant chemotherapy and subsequently correlate with clinical outcome. We used these criteria to develop a new clinico-pathological response 
index (the Nottingham Clinico-Pathological Response Index, NPRI) that can predict the clinical outcome for patients who receive neoadjuvant chemotherapy. 


\section{Materials and Methods}

\section{Patients}

427 patients with clinical stage IIA-IIIC disease (T1-4, N0-3, and M0) who completed neoadjuvant chemotherapy for LABC were included in this study and divided into 3 cohorts according to treatment centre and regimen:

1) The training cohort $(n=172)$ were diagnosed at Nottingham University Hospitals (NUH) between 1996 and 2011 and treated with standard anthracycline regimens $(\mathrm{AC})$ in the form of 6 cycles of FEC (5-fluorouracil $500 \mathrm{mg} / \mathrm{m}^{2}$, epirubicin $75-100 \mathrm{mg} / \mathrm{m}^{2}$, cyclophosphamide $500 \mathrm{mg} / \mathrm{m}^{2}$, on day 1 of a 21-day cycle). This cohort was used to characterize the NPRI and to determine cut-off points for prognostic groups (NPRI-PGs) based on DFS and BCSS.

2) The internal validation cohort $(n=130)$ included patients who were treated at the same institution (NUH) between 2002 and 2011 and received a taxane in addition to the AC regimens (AC-T) as part of clinical trial protocols.

3) The external validation cohort $(n=125)$ were treated at the Breast Clinical Trials Unit at Mount Hospital (Perth, Western Australia) between 1999 and 2011 and received $A C$ regimens with or without a taxane.

Detailed patient demographics and clinico-pathological characteristics were prospectively assessed and regularly updated (summarized in supplementary online table S1). All patients underwent neoadjuvant chemotherapy, mastectomy or breastconserving surgery and axillary dissection, followed by adjuvant radiation therapy. Patients with hormone receptor-positive breast cancer were offered 5 years of adjuvant endocrine therapy. The median follow-up time was 60 months for the entire 
population and all patients gave their informed consent before initiation of therapy. The Nottingham Research Ethics Committee approved this work.

\section{Pathological Review}

Six authors (IOE, SEP, AHSL, BL, DP and TM A-F) contributed to a comprehensive review of the pathology reports and haematoxylin and eosin stained slides from pairmatched diagnostic core biopsies and surgical resection specimens (breast and regional lymph nodes). Pathological features were assessed and their evaluation criteria are summarized in supplementary online table (S2). Absence of fibrosis with or without granulation tissue, and necrosis in both the tumour bed and dissected regional lymph nodes, was considered as evidence for the absence of any pathological response reaction to chemotherapy. pCR was defined as the absence of residual invasive carcinoma in both the breast and regional lymph nodes. The number of histologically positive lymph nodes was determined by examination of serial macroscopic sections of each lymph node. On average, 16 breast-blocks and all submitted lymph nodes were examined for each case before a diagnosis of pCR was reached.

Oestrogen receptor $(E R)$, progesterone receptor (PR) and human epidermal growth factor receptor 2 (HER2) measurements were available for all patients and reassessed according to the most recent American Society of Clinical Oncology/College of American Pathologists (ASCO/CAP) guidelines ${ }^{14,15}$. The primary tumour size and lymph node stage at the time of diagnosis were determined by physical examination and imaging, including mammography and sonography. Clinical staging of the breast cancer at the time of diagnosis (presenting-TNM stage) and the pathological staging after neoadjuvant chemotherapy (yp-TNM stage) were 
determined using the revised American Joint Committee on Cancer (y-AJCC) staging system for breast cancer ${ }^{16}$.

\section{Residual cancer burden}

Residual cancer burden (RCB) was estimated from routine pathologic sections of the primary breast tumour site and the regional lymph nodes after completion of neoadjuvant chemotherapy according to the MD Anderson Cancer Centre criteria ${ }^{4}$. A calculated RCB index for each patient was generated using the calculation formula at the MD Anderson Cancer Centre's website (http://www3.mdanderson.org/app/medcalc/index.cfm?pagename=jsconvert3) (last accessed $19^{\text {th }}$ June 2014).

\section{Detailed statistical methods}

Statistical analyses were performed using STATISTICA (Stat Soft Ltd, Tulsa, USA) and SPSS (version 17, Chicago, USA). Where appropriate, Pearson's chi-squared; student's t-test and ANOVA tests were used. All tests were two-sided with a 95\% confidence interval $(\mathrm{Cl})$ and a $\mathrm{p}$ value of less than 0.05 was considered to be indicative of statistical significance. Cumulative survival probabilities and 5-year DFS were estimated using the univariate Cox models and the Kaplan-Meier plot method where appropriate, and differences between survival rates were tested for significance using the log-rank test.

\section{Development and calculation of NPRI scores}

After definition of factors that were associated with DFS and BCSS, multivariate Cox proportional hazards models (with backward stepwise exclusion of these factors, 
using a criterion of $p<0.05$ for retention of factors in the model) were used to identify factors that were independently associated with clinical outcomes. The statistical significance of the model was assessed based on the likelihood ratio test. The proportional hazards assumption was tested using both standard log-log plots and by generating Kaplan-Meier survival estimate curves, and observing that the curves did not intersect with each other. Hazard ratios (HRs) for relapse and death risks and 95\% confidence intervals were calculated from the Cox proportional hazards analysis. Subsequently, NPRI scores for both DFS and BCSS were calculated using the summations of $\beta$-coefficient values of the factors/measurements retained in the final model after controlling for both hormonal and chemotherapies.

\section{Determination of NPRI cut-off}

As an NPRI score is a continuous risk, to evaluate its efficiency as a prognostic tool we defined pathological response subgroups associated with a prognostic outcome using specific cut-offs. We determined thresholds to define four NPRI prognostic groups (NPRI-PGs) with distinct prognoses: NPRI-PG1 included those with no traces of residual disease (i.e. those who achieved pCR), NPRI-PG2 included those with good response (near-pCR) and NPRI-PG3 and NPRI-PG4 those with moderate and the poorest responses respectively. To determine the first cut-off point (between NPRI-PG4 and other NPRI score groups), a multivariate Cox regression model was used that included the clinical and demographic covariates and a dichotomous NPRI based on cut-off points selected between the $5 \%$ and the $95 \%$ quartiles of the NPRI

score distribution. The optimal cut-off point was selected as the quartile that maximized the profile log-likelihood of this model. A second cut-off point (between NPRI-PG2 and NPRI-PG3) was determined similarly by maximizing the profile log- 
likelihood of a Cox model that included all clinical covariates and the first dichotomous PRI-score factor (i.e. NPRI-PG4 versus NPRI-PG2/3).

\section{Clinical impact of NPRI and model discrimination}

To assess the potential clinical impact of the NPRI, multivariable analyses using the Cox proportional hazards model were performed with the NPRI score as a continuous variable, and/or a prognostic set of clinical-pathological variables controlling for neoadjuvant chemotherapy regimen and adjuvant chemotherapy.

Receiver operating characteristic $(\mathrm{ROC})$ curves were generated to compare the different prognostic models with or without inclusion of NPRI scores. Logistic fit of low versus high survival category by cumulative hazard (the product of the hazard ratios of each incorporated variable) was performed. Area under the curve (AUC) values was calculated from ROC curves. An AUC of 0.8 or above was considered a good classifier.

Model discrimination was evaluated based on Harrell's concordance index ( $c$ index), which is a generalized area under the receiver operating curve (AUC) for censored observations and is equal to the probability of concordance between the predicted probability of relapse and the relapse outcome ${ }^{17}$. The $c$ index was adjusted for bias using bootstrap resampling with 300 replications. The $\mathrm{Cl}$ for the $c$ index was obtained based on approximate normality using the variance estimate of the unadjusted index.

Fitted polynomial function curves were calculated which summarise the broad relativity between the NPRI value and both 5- and 10-year DFS and BCSS. Fitted polynomial curves were constructed from the raw data by applying median DFS and 
BCSS of each NPRI-PG against the 5 and 10 year DFS and BCSS for each division. Predictions from the curves were compared with the actual values.

Predictive accuracy of NPRI compared with other prognostic clinicopathological factors

To evaluate whether the NPRI-PGs add new independent prognostic information to the revised $y$-AJCC stage, $\mathrm{pCR}$ or residual cancer burden (RCB), we performed separate Kaplan-Meier analyses by NPRI prognostic group within each AJCC stage stratum, RCB classes and non-pCR subgroup. The significance of the additional stratification provided by the NPRI was evaluated based on the log-rank test. 


\section{Results}

Clinico-pathological factors associated with higher risk of relapse and death after FEC chemotherapy in univariate Cox analysis included absence of fibrosis in primary tumour site and regional lymph nodes, presence of lymphovascular invasion (LVI), and increasing number of lymph node metastases. These factors maintained significance as independent predictors for both DFS and BSSS after controlling for adjuvant chemotherapy and other covariates by using multivariate Cox proportional hazards models with backward stepwise exclusion. The percentage of reduction in primary tumour size showed statistical significant association with DFS only (Table 1, supplementary table S3, figure S1). None of the covariates exhibited significant deviations from the proportionality assumption or had time-dependent effects (code system used is summarized in supplementary table S4). Subsequently, the summations of $\beta$ coefficient values produced by the Cox analysis were used to calculate the NPRIs for each patient as follows:

\section{NPRI for DFS (NPRIDFS) =}

Fibrosis status $(0,1) \times 1.2830+L V I$ status $(0,1) \times 0.8431+$ number of positive lymph nodes $(0-43) \times 0.0537+$ percentage of reduction of primary tumour size $(+100 \%$ to $-100 \%) \times-0.008162+$ planned hormonal therapy status $(0,1) x-0.7521$

NPRI for BCSS: (NPRI BCSS) =

Fibrosis status $(0,1) \times 1.618028+$ LVI status $(0,1) \times 1.048666+$ number of positive lymph nodes (0-43) x 0.063750 + planed hormonal therapy status $(0,1) \times-1.093202$ 


\section{The prognostic value of the NPRI compared with prognostic pathological and clinical factors}

We examined the predictive accuracy of the NPRI score compared with other prognostic clinico-pathological factors by performing the Cox proportional hazards univariate and multivariable analyses controlling for neoadjuvant and adjuvant chemotherapy. In the training cohort, patients had almost a three-fold increase in both relapse $\left(\mathrm{HR} 2.83 ; 95 \% \mathrm{Cl}, 2.17\right.$ to $\left.3.68 ; \mathrm{p}=1.1 \times 10^{-14}\right)$ and death $(\mathrm{HR} 2.72$; $95 \% \mathrm{Cl}, 2.02$ to $3.66 ; \mathrm{p}=4.6 \times 10^{-11}$ ) for each unit of increase in the NPRI (supplementary table S3). When the NPRI was included in a multivariate Cox regression model (Figures $1-\mathrm{A}$ and supplementary S2-A), the overall predictive power of the model was significantly improved for both DFS $\left(p=3.79 \times 10^{-13}\right)$ and BCSS $\left(p=3.6 \times 10^{-9}\right)$, and the NPRI was significantly associated with a two-fold increase of the risk of disease recurrence (HR 2.07; $95 \% \mathrm{Cl}, 1.36$ to $3.16 ; p=0.001)$ and death (HR 2.14; $95 \% \mathrm{Cl}, 1.37$ to $3.36 ; \mathrm{p}=0.001)$.

Using univariate analysis, a similar statistically significant association between NPRI and both DFS and BCSS was found in both the internal and external validation cohorts (Supplementary table S3). When the Cox proportional hazards multivariable analysis was repeated in the two validation sets controlling for neoadjuvant and adjuvant chemotherapy, the NPRI score outperformed other pathological and clinical covariates and was associated with both DFS and BCSS. All other pathological and clinical covariates failed to show a consistent association with prognosis (Figures 1, $\mathrm{B} \& \mathrm{C}$ and S2 B\&C).

To assess the contribution of the NPRI toward prediction of response to neoadjuvant chemotherapy, Cox proportional hazards statistical models containing relevant 
pathological and clinical predictors controlling for neoadjuvant and adjuvant chemotherapy were constructed. ROC analyses were performed as follows:

1. RCB score alone.

2. NPRI score alone.

3. A prognostic model including RCB score, yp-TNM, c-TNM, histological grade, HER2, ER, pCR status, age, neoadjuvant and adjuvant chemotherapy without NPRI score.

4. The same prognostic model with NPRI scores.

This demonstrated AUC values for DFS of $0.77,0.85,0.82$ and 0.87 for $1,2,3$, and 4 respectively (Figure 2-A). AUC values for BCSS were $0.71,0.85,0.84$ and 0.88 respectively (Supplementary figure S3-A). These results remained consistent when the analysis was repeated for both internal (Figure 2-B and supplementary figure S3B) and external validation cohorts (Figure 2-C and supplementary figure S3-C).

\section{NPRI identifies distinct prognostic groups of none-pCR patients}

We identified two cut-off points to assign patients with residual disease (non-pCR; or non NPRI-PG1) after FEC treatment into one of three classes: NPRI-PG2 (good prognosis group), NPRI-PG3 (moderate prognostic group), and NPRI-PG4 (poor prognostic group). The first cut-off point (NPRI-PG4 v NPRI-PG2/3) was selected as the 83th percentile (NPRI, 0.91021), and as the 92th percentile (NPRI, 1.87383) for DFS and BCSS, respectively. The second cut-off point (NPRI-PG2 v NPRI-PG3) corresponds to the 64.5 th percentile (NPRI, 0.522257) and to the 53.5th percentile (NPRI, 0.01921) for DFS and BCSS, respectively. The cut-off points defined subgroups of NPRI-PG1 to NPRI-PG4 with increasingly poor prognosis (Figure 3). 
The cumulative incidence estimate of the overall probability of relapse within 5 years was $9 \%$ for the pCR group (NPRI PG1) and 13\% for NPRI-PG2, whereas it was $44 \%$ and $86 \%$ for NPRI-PG3 and NPRI-PG4, respectively. Similarly, the cumulative incidence estimate of the overall probability of death within 5 years was $4 \%$ for the pCR group (NPRI PG1) and 5\% for NPRI-PG2, whereas it was 33\% and $71 \%$ for NPRI-PG3 and NPRI-PG4, respectively.

Applicability of the NPRI was evaluated in both the internal and the external independent cohorts. In both cohorts, NPRI defined groups with increasingly poor 5year prognoses (Figures 3-B\&C). The separation of both the 5-year relapse and death rates were somewhat smaller in the training set than for the internal validation cohort. To explore this furthermore, we combined the poor prognosis groups together (NPRI-PG4 and NPRI-PG3) and compared the 5-year DFS and BCSS with those in the NPRI-PG1 (pCR patients) and found the separation of both the 5-year relapse and death rates were still wider in AC-T cohort vs. FEC-only cohort (21\% for DFS and $12 \%$ for BCSS). The $c$-index of the prognostic model on the internal and external validation cohorts was $0.776(95 \% \mathrm{Cl}, 0.67$ to 0.87$)$ and $0.841(95 \% \mathrm{Cl}$, 0.76 to 0.92$)$.

The Pearson correlation coefficient and fitted polynomial function curves showed that there is an excellent inverse linear correlation between NPRI and both DFS and BCSS. In figure 5 , the fitted polynomial function summarises a broad relationship between the NPRI value and median 5- and 10-year survival. 


\section{NPRI prognostic groups stratify clinical outcome of breast cancer molecular sub-groups}

Subgroup analysis of whole patients confirmed that NPRI is a valid prognostic tool regardless of molecular classes of breast cancer (Figure 4-A-D). Applying the NPRI to the ER positive subgroup demonstrated that $45 \%$ of patients might have poor clinical outcome despite receiving adjuvant hormone therapy after completing neoadjuvant chemotherapy, whilst $38 \%$ of ER negative cancers had an excellent prognosis. Moreover, only $58 \%$ of HER2 overexpression/amplification breast cancers had a favourable outcome despite receiving Herceptin following neoadjuvant therapy. Although patients with triple negative breast cancer did not receive targeted adjuvant therapy after neoadjuvant therapy, our results demonstrated that $42 \%$ of those patients had excellent prognosis.

\section{The NPRI prognostic groups stratify prognoses of TNM stages}

Subgroup analyses of the TNM stage at diagnosis also confirmed the prognostic power of the NPRI to separate clinical TNM stage III cancers into distinct DFS $(p=$ $2.6 \times 10^{-15}$, Figure 4-E) and BCSS prognostic groups $\left(p=4.5 \times 10^{-11}\right.$; data not shown). Results demonstrated that $41 \%$ of those patients achieved excellent prognosis.

In addition we evaluated the contribution of the NPRI prognostic group to the prognostic power of each post-therapy yp-TNM stage group (Figure 4-F-G). Regarding DFS and BCSS, NPRI classified both yp-TNM stage II and stage III patients into three distinct prognostic subgroups ( $\mathrm{ps}<0.001$; Figure 4-F-G). Therefore, NPRI classification appears to add significant prognostic power compared 
with post-treatment pathologic y-AJCC stage, at least for stage II/III tumours that represent $2 / 3$ of our patients.

The NPRI adds significant prognostic power compared with Residual Cancer Burden classes

By application of the NPRI to residual cancer burden (RCB) classes in all patients after pooling three cohorts together; the NPRI improved the prognostic stratification of patients who were designated as RCB classes II ( $n=195$; predicted to have a 5year DFS and BCSS of $67 \%$ and $78 \%$, respectively) and III ( $n=110$; predicted to have a 5 -year DFS and BCSS of $46 \%$ and $63 \%$, respectively). Each class has been stratified into 3 distinctive prognostic groups (Figure 4-H-I, ps<0.0001)). Application of NPRI to RCB class II and III, showed that $17 \%$ of those patients actually experienced excellent prognosis and $17 \%$ of RCB class II had a worse prognosis than that predicted for that class of patients. 


\section{Discussion}

Up to $20 \%$ of breast cancer patients present with locally advanced disease which is associated with a poor prognosis ${ }^{15}$. In those tumours that lack a specific therapeutic target (i.e. ER or HER2), conventional chemotherapy remains the mainstay of systemic therapy. Knowledge of the primary tumour's sensitivity or resistance to neoadjuvant chemotherapy can predict the efficacy of these agents on micrometastatic disease. This information can be used to tailor adjuvant therapy after definitive surgery 4 . However, at the present, there are no effective prognostic tools to guide adjuvant therapy based on response to neoadjuvant chemotherapy. Although the pathological evaluation of tumour response is still the gold standard, the lack of uniform reporting of pathological response remains a problem ${ }^{16}$.

In this study, a comprehensive clinico-pathological evaluation of a cohort of patients who received neoadjuvant $\mathrm{AC} / \mathrm{FEC}$ chemotherapy at a single centre was conducted and the NPRI was developed, which was then validated in two independent cohorts. Patients with higher NPRI scores showed statistically significant associations with shorter DFS and BCSS. The NPRI can categorise patients into four prognostic groups (NPRI-PG1 to PG4) with patients in NPRI-PG2 having the same 5-year outcome as those with pCR (NPRI-PG1), irrespective of the type of neoadjuvant chemotherapy administered, ER status, or the pathological stage of residual disease. The prognostic information described herein represents the most detailed data available on DFS and BCSS outcomes for patients treated with neoadjuvant chemotherapy. The NPRI outperforms other traditional predictors of clinical outcome of breast cancer such as residual cancer burden, pCR and the revised yp-TNM 
stage, with high predictive accuracy in the training cohort and in the two independent validation sets.

Application of the NPRI can be used to tailor adjuvant treatment for patients with locally advanced breast cancer. For example, the NPRI identifies a second good prognostic group (NPRI-PG2) that have responded as well as the cohort with pCR (PG1) and can be spared further multiple cycles of unnecessary (and potentially toxic) treatments. Likewise, NPRI-PG3 and PG4 of ER positive patients had a moderate to poor prognosis despite receiving adjuvant hormone therapy after completing neoadjuvant chemotherapy. Therefore, the NPRI identifies an important subset of patients with combined insensitivity to chemo- and hormone therapies that may benefit from novel therapy in a trial setting.

The separation of the 5-year relapse and death rates were somewhat better in patients who received $A C+T$ chemotherapy compared with those who received $A C$ only, indicating some benefit from the addition of a taxane. But this is uncertain due to the lower number of patients in the NPRI-PG4 subgroup. However; after combining NPRI-PG3 and NPRI-PG4 we found the same separation, confirming that those patients might benefit from adding a taxane to anthracycline chemotherapy, in agreement with some published clinical trials ${ }^{17}$. Moreover, the $c$-index of the prognostic model on the internal and external validation cohorts showed similar sensitivity and specificity.

It has been recommended that the predictive ability of a new score should be evaluated based on whether the score improves an already optimized multivariate model of available risk factors ${ }^{18}$. Based on this, a statistical prognostic model, 
including an NPRI score and known prognostic factors, has shown to be superior to all the prognostic models without the NPRI.

Several studies have attempted to provide criteria for response after neoadjuvant chemotherapy $^{16}$ by using clinical, MRI or sonography findings ${ }^{19,20}$ or by bidimensional measurement of the primary tumour bed in resection specimens ${ }^{3,16}$. In fact, these systems have incorporated macro-anatomical features of breast cancer (viz. residual tumour size and lymph node status]. The NPRI, with inclusion of lymphovascular invasion, host response and changes in tumour size, highlights also the importance of the tumour micro environment as a predictor for response to chemotherapy.

In agreement with other studies $3,21,22$ we found lymph node status after neoadjuvant chemotherapy is still the single most important prognostic factor. However, the increasing use of sentinel lymph node biopsy either before or after neoadjuvant chemotherapy leads to difficulties in evaluating the prognostic importance of lymph node status. With regard to the interpretation of sentinel lymph node status after neoadjuvant chemotherapy, the current data is inconsistent and requires further evaluation ${ }^{23}$. However, it is possible that with adequate standardization of the techniques and data from prospective clinical trials, sentinel lymph node status after neoadjuvant chemotherapy could be added to our index for patient selection to reduce surgical morbidity in the good prognostic groups.

In our study, the presence of lymphovascular invasion after neoadjuvant chemotherapy was an independent predictor of clinical outcomes, in agreement with previous studies 24,25 . It has been shown that tumour emboli in vascular spaces are relatively resistant to treatment when compared to carcinoma invading the stroma ${ }^{26}$. 
It should be noted that the identification of lymphovascular invasion may sometimes be difficult as the residual tumour nests or DCIS may show marked retraction artefact in the fibrous stroma mimicking invasion ${ }^{5}$. Thus, proper tissue fixation and immunohistochemical staining for lymphatic channel markers may be useful to distinguish tissue retraction from lymphatic invasion.

Although residual tumour size has been proposed as a prognostic factor for breast cancer ${ }^{10,25}$, we found the reduction in primary tumour size was more predictive of DFS than actual residual tumour size after neoadjuvant chemotherapy. In fact, using residual tumour size does not discriminate between large contiguous carcinomas (which have shown minimal treatment effect) with microscopic foci scattered in a tumour bed with equally great dimensions (demonstrating significant treatment effect). Many examinations have been proposed to monitor the extent of the residual disease extent during neoadjuvant chemotherapy, such as physical examination, mammography and sonography, but most studies demonstrate modest accuracy when compared with final pathological assessment ${ }^{27}$. More recently, there is increasing evidence that magnetic resonance imaging (MRI) is an excellent imaging tool with high specificity rate for both early response monitoring and the assessment of the extent of residual disease ${ }^{28}$. However, relatively few studies reported direct comparisons between MRI and other cost-effective tests ${ }^{28}$. Partridge et $a^{R 9}$ found that MRI tumour volume was more predictive of DFS than tumour diameter, suggesting that volumetric changes measured using MRI may provide a more sensitive assessment of treatment efficacy. Furthermore, changes of metabolic volume measured by functional MRI could reflect early outcomes of neoadjuvant chemotherapy ${ }^{30}$. In fact, future incorporation of MRI results into the NPRI score 
could add another dimension to NPRI for early assessment of response to neoadjuvant chemotherapy even before completion of the full course.

\section{Limitations of the study and future directions}

Although we validated our findings in two independent cohorts and we have shown that the NPRI can supplement existing methods to define pathologic response, the utility of the NPRI requires further validation in larger patient populations. It would require prospective evaluation to demonstrate its role as a prognostic tool and potentially to select patients for novel systemic therapies following neoadjuvant chemotherapy. In addition, further studies are needed to address inter-observer variability, standardization of NPRI measurements and develop a more objective methodology to quantify such factors as fibrosis, lymphovascular invasion and changes in tumour size. Further refinement of the scoring system through the addition of new molecular or biologic markers is also needed.

\section{Conclusion}

We suggest that the incorporation of the NPRI in assessing tumour response following neoadjuvant chemotherapy, will aid in individualising systemic treatment in patients with locally advanced breast cancer, in order to optimize patient outcomes. In particular, it may identify patients who fail to benefit from standard chemotherapy regimens, such that further treatment with novel therapies is warranted. We believe that this scoring system may provide a standardized approach to reporting the tumour response to neoadjuvant chemotherapy. 


\section{Abbreviations}

$\mathrm{AC}=$ Anthracycline and Cyclophosphamide combination

AC-T = Anthracycline and Cyclophosphamide combination, followed by Taxane

AT = Adjuvant Therapy

$\mathrm{AUC}=$ Area Under Curve

$\mathrm{BC}=$ Breast Cancer

BCSS = Breast Cancer Specific Survival

$\mathrm{Cl}=$ Confidence Interval

DCIS = Ductal Carcinoma in Situ

DFS $=$ Disease Free survival

DP $=$ Disease Progression

$E R=$ Oestrogen Receptor

FEC = 5-Fluorouracil (5-FU) $500 \mathrm{mg} / \mathrm{m}^{2}$, Epirubicin 75-100 mg/m², Cyclophosphamide 500 $\mathrm{mg} / \mathrm{m}^{2}$, on day 1 of a 21 -day cycle.

HER2 = Human Epidermal Receptor 2

inv-CA size $=$ Residual invasive size

LABC $=$ Locally Advanced Breast Cancer

LN = Lymph node

LVI = Lympho-vascular Invasion

Neo-ACT = Neoadjuvant chemotherapy 
OS = Overall Survival

$\mathrm{NPRI}=$ Nottingham clinico-Pathological Response Index

$\mathrm{NUH}=$ Nottingham University Hospitals Trust

PER-BC = Primary Oestrogen Receptor Negative Breast Cancer

pCR $=$ Pathological Complete Response (Primary and Lymph nodes are negative)

pre-PTS = pre-chemo Patient Tumour Size (maximum diameter)

PGs = Prognostic Groups

PTS = Patient Tumour Size (maximum diameter)

$\mathrm{RCB}=$ Residual Cancer Burden

$\mathrm{RD}=$ Residual Disease

ROC $=$ Receiver Operating Characteristic curves

$\mathrm{RT}=$ Residual Tumour

SLN = Sentinel Lymph node

$\mathrm{T}=$ Taxane

c-TNM = Clinical TMA stage

$\mathrm{yp}-\mathrm{TNM}=$ revised pathological TNM stage

$\% \mathrm{CA}=$ Percentage of overall cancer cellularity

$\%$ CIS = Percentage of cancer that is residual intra-ductal carcinoma

$\%$ inv-CA $=$ Percentage of invasive component 
$\% \mathrm{cp}$-PTS-R $=$ Percentage of the clinico-pathological tumour size reduction 


\section{Funding}

This work was supported by the Nottingham University Hospitals NHS Trust (NUH) Research and Innovation (R\&I) and Breast Cancer Research Charitable Fund. 


\section{Authors' Contributions}

S.Y.T.C., T.M.A.A-F., G.B. and I.O.E. designed the study. S.Y.T.C., T. M.A.A-F., G.B., A.H.S.L., S.P., R.D.M., E.C., P.M.M., R.S., B.L., D.P., A.C. and I.O.E. were involved in drafting the manuscript, and took part in critically reviewing it for publication. S.Y.T.C., T.M.A.A-F., G.R.B., and I.O.E. analysed and interpreted the data. T.M.A.A-F. undertook the pathological assessment of experimental slides. P.M.M. conducted collection and management of patient data. Figures, tables and referencing

were

generated

by

T.M.A.A-F. 


\section{References}

1. Mauri D, Pavlidis N, loannidis JPA: Neoadjuvant Versus Adjuvant Systemic Treatment in Breast Cancer: A Meta-Analysis. Journal of the National Cancer Institute 97:188-194, 2005

2. Thompson AM, Moulder-Thompson SL: Neoadjuvant treatment of breast cancer. Annals of Oncology 23:x231-x236, 2012

3. Symmans WF, Peintinger F, Hatzis $C$, et al: Measurement of residual breast cancer burden to predict survival after neoadjuvant chemotherapy. J Clin Oncol 25:4414-22, 2007

4. Gralow JR, Burstein HJ, Wood W, et al: Preoperative Therapy in Invasive Breast Cancer: Pathologic Assessment and Systemic Therapy Issues in Operable Disease. Journal of Clinical Oncology 26:814-819, 2008

5. Fan F: Evaluation and reporting of breast cancer after neoadjuvant chemotherapy. OPJ 3:58-63, 2009

6. von Minckwitz G, Untch M, Blohmer J-U, et al: Definition and impact of pathologic complete response on prognosis after neoadjuvant chemotherapy in various intrinsic breast cancer subtypes. Journal of Clinical Oncology 30:1796-1804, 2012

7. Schott AF, Hayes DF: Defining the Benefits of Neoadjuvant Chemotherapy for Breast Cancer. Journal of Clinical Oncology 30:1747-1749, 2012

8. Martin ST, Heneghan HM, Winter DC: Systematic review and meta-analysis of outcomes following pathological complete response to neoadjuvant chemoradiotherapy for rectal cancer. British Journal of Surgery 99:918-928, 2012

9. Rastogi $P$, Anderson SJ, Bear HD, et al: Preoperative Chemotherapy: Updates of National Surgical Adjuvant Breast and Bowel Project Protocols B-18 and B-27. Journal of Clinical Oncology 26:778-785, 2008

10. Debled M, Mauriac L: Neoadjuvant chemotherapy: are we barking up the right tree? Annals of Oncology 21:675-679, 2010

11. Wolff AC, Hammond ME, Schwartz JN, et al: American Society of Clinical Oncology/College of American Pathologists guideline recommendations for human epidermal growth factor receptor 2 testing in breast cancer. J Clin Oncol 25:118-45, 2007

12. Hammond ME, Hayes DF, Wolff AC, et al: American Society of Clinical Oncology/College of American Pathologists guideline recommendations for immunohistochemical testing of estrogen and progesterone receptors in breast cancer. $\mathrm{J}$ Oncol Pract 6:195-7, 2010

13. Singletary SE, Allred C, Ashley $P$, et al: Revision of the American Joint Committee on Cancer Staging System for Breast Cancer. Journal of Clinical Oncology 20:3628-3636, 2002

14. Harrell JF: Regression modelling strategies: With applications to linear models, logistic regression, and survival analysis. New York, Springer-Verlag 2001

15. Jemal $A$, Ward $E$, Thun M: Recent trends in breast cancer incidence rates by age and tumour characteristics among U.S. women. Breast Cancer Research 9:R28, 2007

16. Sahoo S, Lester SC: Pathology of Breast Carcinomas After Neoadjuvant Chemotherapy: An Overview With Recommendations on Specimen Processing and Reporting. Archives of Pathology \& Laboratory Medicine 133:633-642, 2009

17. Nabholtz J-M, Riva A: Taxane/Anthracycline Combinations: Setting a New Standard in Breast Cancer? The Oncologist 6:5-12, 2001

18. Kattan MW: Judging New Markers by Their Ability to Improve Predictive Accuracy. Journal of the National Cancer Institute 95:634-635, 2003

19. Jeruss JS, Mittendorf EA, Tucker SL, et al: Combined Use of Clinical and Pathologic Staging Variables to Define Outcomes for Breast Cancer Patients Treated With Neoadjuvant Therapy. Journal of Clinical Oncology 26:246-252, 2008

20. Rodenhuis S, Mandjes IAM, Wesseling J, et al: A simple system for grading the response of breast cancer to neoadjuvant chemotherapy. Annals of Oncology 21:481487,2010 
21. Bear HD, Anderson S, Smith RE, et al: Sequential Preoperative or Postoperative Docetaxel Added to Preoperative Doxorubicin Plus Cyclophosphamide for Operable Breast Cancer: National Surgical Adjuvant Breast and Bowel Project Protocol B27. Journal of Clinical Oncology 24:2019-2027, 2006

22. Pinder SE, Provenzano E, Earl H, et al: Laboratory handling and histology reporting of breast specimens from patients who have received neoadjuvant chemotherapy. Histopathology 50:409-417, 2007

23. Lyman GH, Giuliano AE, Somerfield MR, et al: American Society of Clinical Oncology Guideline Recommendations for Sentinel Lymph node Biopsy in Early-Stage Breast Cancer. Journal of Clinical Oncology 23:7703-7720, 2005

24. Katz A, Strom EA, Buchholz TA, et al: The influence of pathologic tumour characteristics on locoregional recurrence rates following mastectomy. International journal of radiation oncology, biology, physics 50:735-742, 2001

25. Chen AM, Meric-Bernstam F, Hunt KK, et al: Breast Conservation After Neoadjuvant Chemotherapy: The M.D. Anderson Cancer Center Experience. Journal of Clinical Oncology 22:2303-2312, 2004

26. Sharkey FE, Addington SL, Fowler LJ, et al: Effects of preoperative chemotherapy on the morphology of resectable breast carcinoma. Mod Pathol 9:893-900, 1996

27. Lobbes $M$, Prevos $R$, Smidt $M$ : Response monitoring of breast cancer patientsreceiving neoadjuvant chemotherapy using breast MRI - a review of current knowledge. journal of Cancer Therapeutics and Research 1, 2012

28. Marinovich ML, Macaskill P, Irwig L, et al: Meta-analysis of agreement between MRI and pathologic breast tumour size after neoadjuvant chemotherapy. $\mathrm{Br} J$ Cancer 109:1528-1536, 2013

29. Partridge SC, Gibbs JE, Lu Y, et al: MRI Measurements of Breast Tumour Volume Predict Response to Neoadjuvant Chemotherapy and Recurrence-Free Survival. American Journal of Roentgenology 184:1774-1781, 2005

30. Chen J-H, Su M-Y: Clinical Application of Magnetic Resonance Imaging in Management of Breast Cancer Patients Receiving Neoadjuvant Chemotherapy. BioMed Research International 2013:14, 2013 
Table (1): Univariate and multivariate backward step-wise analysis for factors associated with disease free survival (DFS) in the training cohort.

\begin{tabular}{|c|c|c|c|c|c|c|c|c|}
\hline \multirow{3}{*}{\begin{tabular}{|l|} 
Risk Factors \\
Age (continuous variable) \\
\end{tabular}} & \multicolumn{4}{|c|}{ Univariate analysis } & \multicolumn{4}{|c|}{ Multivariate analysis } \\
\hline & \multirow{2}{*}{\begin{tabular}{|c|} 
HR \\
1.03
\end{tabular}} & \multicolumn{2}{|c|}{$95 \% \mathrm{Cl}$} & \multirow{2}{*}{$\begin{array}{c}p \\
0.005 \\
\end{array}$} & \multirow[t]{2}{*}{ HR } & \multicolumn{2}{|c|}{$95 \% \mathrm{Cl}$} & \multirow[t]{2}{*}{$p$} \\
\hline & & 1.01 & 1.06 & & & & & \\
\hline Hormonal therapy (Yes, No) & 0.74 & 0.43 & 1.26 & 0.265 & 0.45 & 0.25 & 0.81 & 0.008 \\
\hline Adjuvant Chemotherapy (Yes, No) & 1.56 & 0.89 & 2.74 & 0.118 & & & & \\
\hline Presenting clinical tumour size & 1.01 & 1.00 & 1.02 & 0.003 & & & & \\
\hline Presenting histological grade (High) & 0.91 & 0.53 & 1.57 & 0.744 & & & & \\
\hline Tumour type other than IDC-NST & 1.47 & 0.76 & 2.86 & 0.253 & & & & \\
\hline ER expression (negative) & 1.05 & 0.62 & 1.80 & 0.852 & & & & \\
\hline HER2 status (overexpression) & 0.76 & 0.37 & 1.57 & 0.463 & & & & \\
\hline PR expression (negative) & 0.94 & 0.51 & 1.72 & 0.837 & & & & \\
\hline Triple negative phenotype (Yes, No) & 0.96 & 0.54 & 1.71 & 0.894 & & & & \\
\hline 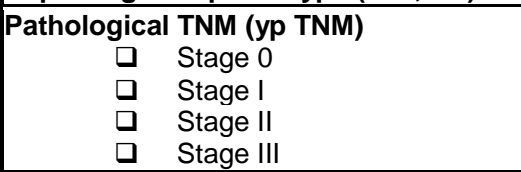 & $\begin{array}{l}1.00 \\
0.78 \\
3.41 \\
7.05\end{array}$ & $\begin{array}{l}0.13 \\
1.00 \\
2.15\end{array}$ & $\begin{array}{l}4.67 \\
11.6 \\
23.1\end{array}$ & $1.3 \times 10^{-4}$ & & & & \\
\hline Residual inv CA size & 1.02 & 1.01 & 1.02 & $1.5 \times 10^{-5}$ & & & & \\
\hline Tumour size reduction & 0.26 & 0.13 & 0.54 & $2.6 \times 10^{-4}$ & 0.99 & 0.98 & 1.00 & 0.022 \\
\hline Number of positive lymph nodes & 1.06 & 1.03 & 1.09 & $1.6 \times 10^{-4}$ & 1.05 & 1.01 & 0.09 & 0.013 \\
\hline Percentage of residual inv CA & 1.02 & 1.01 & 1.03 & $1.0 \times 10^{-5}$ & & & & \\
\hline $\begin{array}{c}\text { Extension and distribution of Inv CA } \\
\square \text { No invasive/scattered cells } \\
\square \text { One focus or scattered foci } \\
\square \text { Nests, groups or sheets }\end{array}$ & $\begin{array}{l}1.00 \\
2.40 \\
5.50\end{array}$ & $\begin{array}{l}0.93 \\
5.51\end{array}$ & $\begin{array}{c}6.33 \\
14.55\end{array}$ & $4.6 \times 10^{--4}$ & & & & \\
\hline Absence of fibrosis & 4.14 & 2.40 & 7.16 & $4.0 \times 10^{-7}$ & 3.65 & 2.07 & 6.45 & $8.0 \times 10^{-6}$ \\
\hline Presence of LVI & 3.04 & 1.77 & 5.23 & $6 \times 10^{-5}$ & 2.17 & 1.20 & 3.89 & 0.010 \\
\hline Presence of ductal carcinoma in situ & 1.60 & 0.93 & 2.86 & 0.088 & & & & \\
\hline
\end{tabular}

IDC-NS; invasive ductal carcinoma-no special type, ER; oestrogen receptor, PR; progesterone receptor, HER2; human epidermal receptor 2, inv-CA; invasive carcinoma, LVI; Lymphovascular invasion 


\section{Figure legends}

Figure 1: A-C. Multivariable Cox proportional hazards regression analyses for disease free survival (DFS; left panel) and corresponding forest plots (Right panel). Comparison of Nottingham clinico-pathological response index (NPRI) score (as continuous variable) with known prognostic clinico-pathological factors including: pathological complete response ( $p C R$ vs. non-pCR), residual cancer burden (RCB) score, presenting clinical TNM (Tumour, Node and Metastases) stage (II vs. III), revised pathological TNM stage (yp-TNM; stage $0 \mathrm{vs.} \mathrm{I/II/III),} \mathrm{histological} \mathrm{grade}$ based on Nottingham grading system (1 vs. 2/3), ER (oestrogen receptor) expression status (negative vs. positive), HER2 (human epidermal receptor 2) overexpression/amplification status (overexpression/amplification vs. no overexpression/amplification), adjuvant chemotherapy, adjuvant hormonal therapy, neoadjuvant therapy (if applicable) and age at diagnosis ( $\leq 54$ vs. $\geq 55$ years) in the training (A), internal validation (B) and external validation (C) cohorts. Solid squares represent the hazard ratio (HR) of recurrence and open-ended horizontal lines represent the $95 \%$ confidence intervals (Cls). All $p$ values were calculated using Cox proportional hazards analysis and $p<0.05$ was considered as statistical significant $p$ value. AC: Anthracycline, T: Taxane, AC-T: Anthracycline and Taxane.

Figure 2: Receiver operating characteristic (ROC) analysis of Nottingham clinicopathological response index (NPRI) score and other clinico-pathological covariates were performed for predicting disease free survival in the training $(\mathbf{A})$, internal validation (B) and external validation (C) cohorts. The area under the curve (AUC) was calculated for ROC curves, and sensitivity and specificity was calculated to assess the performance of residual cancer burden (RCB) alone (1), NPRI alone (2), and ${ }^{*}$ a statistical prognostic model that constructed based on multivariable Cox proportional hazards incorporating known clinico-pathological prognostic variables including: pathological complete response (pCR), RCB score, presenting clinical TNM (Tumour, Node and Metastases) stage, revised pathological TNM stage (ypTNM) stage, histological grade based on Nottingham grading system, ER (oestrogen receptor) expression status, HER2 (human epidermal receptor-2) status, and age at diagnosis (3). ** ROC analysis was also performed for the aforementioned prognostic model after incorporating the NPRI score (4). Dashed grey lines indicate the $45^{\circ}$ angle tangent line marked at a point that provides best discrimination between true positives and false positives, assuming that false positives and false negatives have similar costs. AC: Anthracycline, T: Taxane, AC-T: Anthracycline and Taxane.

Figure 3: Kaplan Meier curves and lifetime table showing disease free survival (upper panel) and breast cancer specific survival (lower panel) in the training (A), internal validation (B), and external validation (C) cohorts stratified according to Nottingham clinicopathological response index- prognostic groups (NPRI-PGs). See text for details. AC: Anthracycline, T: Taxane, AC-T: Anthracycline and Taxane. 
Figure 4: A-D. A-D. Kaplan Meier curves showing DFS of oestrogen receptor (ER) positive (A), ER-negative (B), HER2 overexpression/amplification (C) and triple negative (D) breast cancer patients, stratified according to NPRI- prognostic groups (NPRI-PGs). E. Kaplan Meier curves showing DFS of presenting clinical TNM stage III stratified according to NPRI-PGs. F. Kaplan Meier curves showing DFS of revised pathological TNM stage II (yp-TNM stage II; F) and yp-TNM stage III (G) patients stratified according to NPRI-PGs. Kaplan Meier curves showing DFS of residual cancer burden (RCB) class II $(\mathrm{H})$ and class III (I) patients in the training cohort stratified according to NPRI-PGs. See text for details. AC: Anthracycline, T: Taxane, AC-T: Anthracycline and Taxane.

Figure 5: Fitted polynomial function curves and equations for disease free survival (DFS, A) and breast cancer specific survival (BCSS; B) summarises a broad relationship between the Nottingham clinicopathological response index (NPRI) value and median 5 (dashed line) and 10 (solid line) year survivals in the training cohort. 


\section{Supplementary online figure legends}

Supplementary Figure S1: Kaplan Meier curves showing disease free survival (DFS) in the training (upper panels), internal validation (middle panels) and external validation (lower panels) cohorts stratified according to number of lymph node (LN) metastases (i), presence of lympho-vascular invasion (LVI; ii), absence of fibrosis (iii) percentage of reduction in primary tumour size (iv). See text for details. AC: Anthracycline, T: Taxane, AC-T: Anthracycline and Taxane.

Supplementary Figure S2: A-C. Multivariable Cox proportional hazards regression analyses for breast cancer specific survival (BCSS; left panel) and corresponding forest plots (Right panel). Comparison of Nottingham clinico-pathological response index (NPRI) score (as a continuous variable) with known prognostic clinicopathological factors including: pathological complete response ( $\mathrm{pCR}$ vs. non-pCR), residual cancer burden (RCB) score, presenting clinical TNM (Tumour, Node and Metastases) stage (II vs. III), revised pathological TNM stage (yp-TNM; stage 0 vs. I/II/III), histological grade based on Nottingham grading system (1 vs. 2/3), ER (oestrogen receptor) expression status (negative vs. positive), HER2 (human epidermal receptor 2) overexpression/amplification status (overexpression/amplification vs. no overexpression/amplification), adjuvant chemotherapy, adjuvant hormonal therapy, neoadjuvant therapy (if applicable) and age at diagnosis ( $\leq 54$ vs. $\geq 55$ years) in the training (A), internal validation (B) and external validation (C) cohorts. Solid squares represent the hazard ratio (HR) of recurrence and open-ended horizontal lines represent the 95\% confidence intervals (Cls). All $p$ values were calculated using Cox proportional hazards analysis and $p<$ 0.05 was considered a statistical significant $p$ value. AC: Anthracycline, T: Taxane, AC-T: Anthracycline and Taxane.

Supplementary Figure S3: Receiver operating characteristic (ROC) analysis of Nottingham clinico-pathological response index (NPRI) score and other clinicopathological covariates were performed for predicting breast cancer specific survival in the training $(\mathbf{A})$, internal validation $(\mathbf{B})$ and external validation $(\mathbf{C})$ cohorts. The area under the curve (AUC) was calculated for ROC curves, and sensitivity and specificity was calculated to assess the performance of residual cancer burden (RCB) alone (1), NPRI alone (2), and * a statistical prognostic model that constructed based on multivariable Cox proportional hazards incorporating known clinicopathological prognostic variables including: pathological complete response (pCR), RCB score, presenting clinical TNM (Tumour, Node and Metastases) stage, revised pathological TNM stage (yp-TNM) stage, histological grade based on Nottingham grading system, ER (oestrogen receptor) expression status, HER2 (human epidermal receptor-2) status, and age at diagnosis (3). ${ }^{* *} \mathrm{ROC}$ analysis was also performed for the aforementioned prognostic model after incorporating the NPRI score (4). Dashed grey lines indicate the $45^{\circ}$ angle tangent line marked at a point that provides best discrimination between true positives and false positives, assuming that false positives and false negatives have similar costs. AC: Anthracycline, T: Taxane, AC-T: Anthracycline and Taxane. 